\title{
Real-Time Decision-Making Systems: Approaches to Construction
}

\author{
Zakharchenko I., Dmitriev A., Ovcharenko V., Maslov I., Pavlenko M., Timochko A., \\ Kryzhevska K. \\ Kharkiv National Air Force University named after Ivan Kozhedub \\ Kharkiv, Ukraine
}

\begin{abstract}
The purpose of this work is the development of mathematical tools for formalizing decisionmaking problems in open expert real-time control systems. The goal was achieved by defining and formally describing all the elements of a formal system. The most significant result was the proposed approach to formalization. With its help, within the framework of a single formalism, the dynamic properties of the subject area and the logical-analytical activity of the power system dispatcher, presented in different classes of formal logics, were described. The significance of the results obtained lies in the possibility of a rigorous description of various aspects of knowledge within the framework of a single formal apparatus with further pragmatic interpretation in the management process. The proposed approach was distinguished by using the axioms of aletic and deontic logics and the development of axioms that reflect the specifics of the problems being solved. The introduced system of basic concepts and relations makes it possible to classify many decision-making problems for the power systems management. The goals were described within the framework of a single formalism form the basis of the apparatus for formalizing the decision-making problems of the class under consideration. The formalization apparatus provides a description of the dynamic properties of the system within each aspect of knowledge of the content paradigm. The direction of further research is the construction of an appropriate formal theory based on the proposed formal system.
\end{abstract}

Keywords: control system, decision-making system, power system, real time, target setting, formal system.

DOI: https://doi.org/10.52254/1857-0070.2022.1-53.10

UDC: $621.311: 004.584$

\author{
Sisteme decizionale în timp real: abordări pentru proiectare \\ Zakharchenko I.V., Dmitriev A.G., Ovcharenko V.V., Maslov I.Z., Pavlenko M.A., Timochko A.I., \\ Kryzhevskaya E.V. \\ Universitatea Națională a Forțelor Aeriene din Harkov Ivan Kozhedub \\ Harkov, Ucraina
}

Rezumat. Scopul lucrării este de a elabora un set de instrumente matematice pentru formalizarea sarcinilor de luare a deciziilor în sistemele expert deschise de management în timp real. Obiectivul este atins prin definirea și descrierea formală a tuturor elementelor sistemului formal. Cel mai semnificativ rezultat este abordarea propusă pentru formalizare, prin care, în cadrul unui formalism unic sunt descrise proprietățile dinamice ale domeniului și activităţile logico-analitice ale dispecerului sistemului energetic, reprezentate în diferite clase de logici formale. Semnificația rezultatelor obținute constă în posibilitatea descrierii stricte a diferitelor aspecte ale cunoștințelor în cadrul aparatului formal unificat, cu o interpretare pragmatică ulterioară în procesul de management. În principiu, abordarea propusă diferă prin aplicarea axiomelor logicii aletice și deontice ca logici și dezvoltarea unor axiome care reflectă specificul sarcinilor de rezolvat. Acestea fac legătura între caracteristicile situației și descrierea formalizată a proceselor relevante din domeniul problemei. Totalitatea axiomelor avute în vedere ne permite să reprezentăm procesul de realizare a obiectivelor, fără a fi legați de conținutul specific al problemelor de management care trebuie rezolvate. Această soluție ne permite să considerăm totalitatea axiomelor propuse ca fiind axiome ale pragmaticii teleotice. $\mathrm{Cu}$ ajutorul sistemului introdus de concepte și relații de bază, este posibilă clasificarea întregii mulţimi de sarcini de luare a deciziilor pentru dirigarea sistemului energetic. Obiectivele, descrise în cadrul formalismului unificat, constituie baza aparatului de formalizare a sarcinilor de decizie ale clasei respective. Particularitățile domeniului determină punerea în aplicare a următoarelor tipuri de relații: subordonare, precedență și "condiție inițială - rezultat".

Cuvinte-cheie: sistem de control, sistem decizional, sistem energetic, timp real, stabilirea obiectivelor, sistem formal.

\footnotetext{
(C) Захарченко И.В., Дмитриев А.Г., Овчаренко В.В., Маслов И.З., Павленко М.А., Тимочко А.И., Крыжевская Е.В., 2022
} 


\section{Системы принятия решений реального времени: подходы к построению Захарченко И.В., Дмитриев А.Г., Овчаренко В.В., Маслов И.З., Павленко М.А., Тимочко А.И., Крыжевская Е.В. \\ Харьковский национальный университет Воздушных Сил имени Ивана Кожедуба Харьков, Украина}

Аннотация. Цель работы - разработка математического инструментария формализации задач принятия решений в открытых экспертных системах управления реального времени. Поставленная цель достигается путем определения и формального описания всех элементов формальной системы. Наиболее существенным результатом является предложенный подход к формализации, с помощью которого в рамках единого формализма описываются динамические свойства предметной области и логикоаналитическая деятельность диспетчера энергосистемы, представленные в разных классах формальных логик. Значимость полученных результатов состоит в возможности строгого описания различных аспектов знаний в рамках единого формального аппарата с дальнейшей прагматической интерпретацией в процессе управления. Принципиально предлагаемый подход отличается применением аксиом алетической и деонтической логик в качестве логических и разработкой аксиом, отражающих специфику решаемых задач. Они связывают признаки ситуации и формализованное описание соответствующих процессов в проблемной области. Вся совокупность рассматриваемых аксиом позволяет представить процесс достижения целей, не привязываясь к конкретному содержанию решаемых задач управления. Такое решение позволяет рассматривать совокупность предложенных аксиом как аксиомы телеотической прагматики. С помощью введенной системы базовых понятий и отношений возможно классифицировать все множество задач принятия решений для управления энергосистемами. Цели, описываемые в рамках единого формализма, составляют основу аппарата формализации задач принятия решений рассматриваемого класса. Особенности предметной области обуславливают реализацию следующих видов отношений: подчинения, предшествования и “начальное условие - результат". Аппарат формализации позволяет установить однозначное соответствие между процессами актуализации и достижения целевых установок и фактом их достижения в объективной реальности. Обеспечивается описание динамических свойств системы управления в рамках каждого аспекта знаний содержательной парадигмы. Направлением дальнейших исследований является построение соответствующей формальной теории на основе предложенной формальной системы.

Ключевые слова: система управления, система принятия решений, энергосистема, реальное время, целевая установка, формальная система.

\section{ВВЕДЕНИЕ}

Энергосистемы относятся к одной из критических областей инфраструктуры, управление которой осуществляется в масштабе реального времени (в темпе процесса управления). Текущее состояние энергосистемы определяет стратегию оперативного управления ею и принятие соответствующих решений.

Уровень развития систем управления и подготовки диспетчеров являются основой надежной работы энергосистем, особенно в аварийных ситуациях. Навыки, полученные диспетчерами в процессе длительной работы, фактически являются искусством при диспетчерском управлении энергосистемами.

Поэтому значительная роль в обеспечении надежной работы энергосистем отводится советчикам (системам поддержки принятия решений (СППР)) диспетчера. Для ликвидации аварийных ситуаций диспетчер обязан распределить энергосистемы на несинхронно работающие части и погасить отдельные районы. А далее он должен установить очередность восстановления питания погашенных районов, определить порядок синхронизации отдельных частей, подать напряжение на сборные шины АЭС для надежного питания собственных нужд и обеспечить нагрузку на выделившиеся блоки АЭС и ГРЭС.

Даже опытные диспетчеры при построении адекватного представления об аварии на энергетических объектах часто принимают не оптимальные решения. Это обусловлено необходимостью [1-2]:

- работы в масштабе реального времени;

- анализа сложившейся обстановки, обоснования и принятия решений в условиях острого дефицита времени;

- работы с большими объемами неполной и частично недостоверной информации;

- достоверного и оперативного прогнозирования развития ситуации и выработки эффективных контрмер для ликвидации аварийных режимов; 
- учитывать процедуры логикоаналитической деятельности диспетчеров, характерные для данной предметной области.

Фактически встал вопрос об эффективном использовании знаний путем создания ситуационных алгоритмов на этапе оперативного управления. Решение этой задачи возможно на основе экспертных систем [1]. Однако традиционные экспертные системы из-за изолированности комплексов программ не позволяют решать задачи данного класса.

А отсутствие формальной системы для описания с единых позиций динамических свойств рассматриваемой предметной области и процедур логико-аналитической деятельности диспетчеров сдерживает развитие соответствующих СППР.

Как правило, в любой предметной области предложенные ситуационные алгоритмы охватывают значительный (но не весь!) класс состояний. Это характерно и для описания аварийных состояний энергосистем. В общем случае возможно расширение класса аварийных состояний и, следовательно, построение новых сопутствующих им ситуационных алгоритмов. Это свидетельствует об открытости предлагаемой экспертной системы, которая функционирует в масштабе реального времени.

Таким образом, в науке и практике имеется противоречие. С одной стороны, необходимо автоматизировать процесс принятия решений, происходящий в масштабе реального времени. С другой стороны, невозможно удовлетворить этим требованиям при использовании существующих средств автоматизации управления. Данное противоречие может быть разрешено путем совершенствования теории открытых экспертных систем реального времени. Автоматизация задач принятия решений, которые необходимо решать в масштабе реального времени, является перспективным направлением в теории автоматизации управления.

\section{Анализ литературы}

Вопросы автоматизации выработки и принятии решений в системах управления на объектах энергетической сферы рассмотрены в работах $[1,2]$. Показана принципиальная невозможность применения традиционных экспертных систем для решения задач данного класса.
Особенности применения моделей знаний основанных на логике предикатов рассмотрены в источниках $[3,4]$ для удовлетворения требованиям, предъявляемым к средствам формализации и принятия решений по управлению на объектах энергетики. В работе [3], в частности, уделено внимание вопросам корректности соответствующих формализованных описаний.

Рассмотрение вопросов корректности процесса принятия решений для конкретных cфер применения с использованием структуризации и упорядочения представлено в тркдах [5-9]. Не рассмотрено определение отношений, характеризующих связность пар целей в иерархической системе; отношение необходимости и достаточности достижения каждой цели рассматривается отдельно.

Публикация [10] посвящена постановке задачи принятия решений в сложных обстоятельствах. Однако она осуществлена в общем виде, не учитывающем особенности предметной области.

В работе [11] не доказана необходимость однозначного соответствия между реальными процесами и моделируемыми с помощю апарата формализации.

Модель приведенная в [12] не учитывает в полном объеме вопросы полноты и строгости отношений для открытой экспертной системы объектов энергетики.

Вопросы рассмотренные в [13] посвящены изучению отношений подчинения строгого порядка. Однако не рассмотрены вопросы применения этих отношений подчинения для описания пердметной области.

Анализ отношений слабого предшествования рассмотрен в работе [14], но не показано, каким образом отношения слабого предшествования могут быть применены при построении новых ситуационных алгоритмов.

В работе [15] не рассматривается порядок выбора наилучшего в некотором смысле варианта достижения цели из нескольких.

Ограниченное использование формальных систем алетической и деонтической логик в системе модально-временных операторов для решения ряда практических задач приведено в [12-16]. При этом возможность их реализации рассмотрено для ограниченного круга формальных систем. 
Описание динамических свойств в рамках прагматичного аспекта знаний для системы метеонаблюдений приведено в источнике [17], которое требует доработки для системы управления объектами энергетики.

Рассмотрению вопросов применения систем поддержки перинятия решений в различных областях посвященны работы [1824]. Однако для систем управления реального времени необходима разработка аппарата формализации, описывающая разные аспекты знаний в рамках единого формализма.

Работа [25] не отражает особенности управления энергетическими объектами в реальном времени при построении мультиагентной системы принятия решений.

Описание модели G2 хорошо описано в материалах [26]. Предложено решение в виде блоков, связанных "жесткой" последовательностью выполнения операций. Такой подход неприемлем для описания каждый раз уникальных ситуаций, возникающих в процессе функционирования объектов энергетики.

Таким образом, для формализации процесса принятия решений требуется строгое определение понятий цели, отношений между целями и разработка соответствующего математического аппарата. Он позволяет устанавливать истинность отношений между целями по каждому из аспектов знаний, определяющих содержательную парадигму, описывать структуру целевой модели и контролировать процессы достижения целевых установок и пополнения знаний о задачах принятия решений.

Кроме того, интерес представляет широко используемая модель на базе G2 [26]. Однако ее использование для создания открытой экспертной системы для управления в сфере энергетики сопряжено с рядом принципиальных трудностей. И неспроста: среди сфер ее применения нет энергетики. Это связано с тем, что вводимое понятие рабочего объекта модели является динамическим. В процессе моделирования происходит обработка объекта (заказы, расходные материалы, готовые продукты и т. п.). Рабочие объекты передаются в модели по установленным путям и подвергаются некоторым изменениям (преобразованиям). Приоритет операции не устанавливается (в очереди: первым пришел - первым ушел). Однако описание модели в G2 представлено в виде блоков, связанных "жесткой" последовательностью выполнения операций. Такой подход неприемлем для описания каждый раз уникальных ситуаций, возникающих в процессе функционирования объектов энергетики.

\section{МЕТОДЫ, РЕЗУЛЬТАТЫ И ОБСУЖДЕНИЕ}

В общем случае для принятия решения в сложных ситуациях известны перечни проблем $\bar{P}=\left(p_{1}, p_{2}, \ldots, p_{n}\right)$, и ограничений на возможные действия $\bar{O}=\left(o_{1}, o_{2}, \ldots, o_{m}\right)$. Необходимо сформировать множества достижимых целей $\bar{L}=\left(u_{1}, u_{2}, \ldots, u_{k}\right)$, возможных вариантов их достижения $\bar{D}=\left(d_{1}, d_{2}, \ldots, d_{l}\right)$ и выбрать оптимальный в некотором смысле набор вариантов $\bar{D}^{*}=\left(d_{1}^{*}, d_{2}^{*}, \ldots, d_{l}^{*}\right) \quad$ для достижения поставленной цели [10].

Постановка цели всегда связана с осознанием определенной проблемы $P$. Устранение проблемы - это определение возможных действий по ее решению через достижение определенных целей. Возможные альтернативные пути достижения цели строятся так: формулирование цели; построение иерархической системы подцелей; определение состава подцелей для данной проблемы; выбор альтернативных путей для достижения подцелей; поиск оптимальных путей достижения цели.

Пусть цели в сетевой модели выражаются некоторыми формулами. Они отражают алетические и деонтические аспекты знаний о цели и определяют ее характер. Факт достижения цели в ситуации $\alpha$ определяется характеристической функцией $\vec{\phi}(\alpha)$.

Формулировка цели в общем случае может включать несколько логически связанных формул по каждому из аспектов знаний. Пусть цель $а$ представляет собой конъюнкцию формул, образующих множество $\Phi_{a}$. Тогда, если $\left(X \in \Phi_{a}\right)$, то $X$ является конъюнктивной составляющей $a$. Когда формула цели $a$ является дизъюнкцией множества формул, и отрицание формулы $\sim X$ является элементом множества $\left(\sim X \in \Phi_{a}\right)$, то $X$ является дизъюнктивной составляющей цели $a$.

Необходимость достижения цели $N_{D}$ характеризуется деонтическим аспектом 
знаний и определяется соответствующим значением истинности $T_{D}$ или $F_{D}$.

Если существует множество формул $\left\{x_{1}, x_{2}, \ldots, x_{m}, y\right\}$ таких, что необходимость достижения $N_{D} y$ определяет необходимость достижения целей $x_{1} \wedge x_{2} \wedge \ldots \wedge x_{m}$, т.е.

$$
\begin{aligned}
& x_{1} \wedge x_{2} \wedge \ldots \wedge x_{m} \rightarrow y, \\
& y \rightarrow x_{1} \wedge x_{2} \wedge \ldots \wedge x_{m}
\end{aligned}
$$

истинны $\left(T_{D}\right)$ для определенной цели $y$, то цель $у$ является конъюнктивной.

Возможности достижения цели в сложившейся ситуации $\alpha$ и любой гипотетической ситуации характеризуются алетическим аспектом знаний. Поэтому цель достижима, если соответствующая формула имеет значение $T_{A}$.

Если истинными $T_{A}$ для $\left\{x_{1}, x_{2}, \ldots, x_{m}, y\right\}$ являются выражения:

$$
\begin{aligned}
& x_{1} \wedge x_{2} \wedge \ldots \wedge x_{m} \rightarrow M \uparrow y, \\
& M \uparrow y \rightarrow x_{1} \wedge x_{2} \wedge \ldots \wedge x_{m},
\end{aligned}
$$

тогда цель $y$ является конъюнктивно достижимой.

Но $T_{A}$ характеризует только возможность, но не факт достижения цели. Но для некоторой цели истинность конъюнкции формул $x_{1} \wedge x_{2} \wedge \ldots \wedge x_{m} \quad$ является необходимым и достаточным условием. Аналогично определим понятие дизъюнктивной цели для $T_{D}$ i $T_{A}[11]$.

Если истинными $T_{A}$ являются выражения:

$$
\begin{aligned}
& x_{1} \vee x_{2} \vee \ldots \vee x_{m} \rightarrow M \uparrow y, \\
& M \uparrow y \rightarrow x_{1} \vee x_{2} \vee \ldots \vee x_{m},
\end{aligned}
$$

тогда цель называется дизъюнктивно достижимой. Истинность хотя бы одного дизъюнктивного компонента является необходимым и достаточным условием истинности дизъюнктивной цели.

Однако признаков описания целей явно недостаточно.

Необходимость и возможность достижения соответствующих целевых установок (2-8) представлены без их корреляции с реализованными событиями. А требуется однозначное соответствие между смоделированными и реальными процессами во всех точках реализации элементарных управляющих воздействий.

Пусть цели сетевой модели однозначно соответствует характеристическая функция

$$
\begin{aligned}
& \vec{\varphi}(\alpha, t)=\wedge_{i=1}^{n} \phi_{i}(\alpha, t) \equiv \phi_{1}(\alpha, t) \wedge \\
& \wedge \phi_{2}(\alpha, t) \wedge \ldots \wedge \phi_{n}(\alpha, t),
\end{aligned}
$$

где $\alpha$ - ситуационная переменная, описывающая состояние проблемной среды;

$t$ - момент времени;

$$
\phi_{i}(\alpha, t)=\left\{\begin{array}{l}
\varphi_{i}(\alpha, t), \\
\sim \varphi_{i}(\alpha, t),
\end{array}\right.
$$

выражающая состояние предметной области;

$\varphi_{i}(\alpha, t)$ - элементарная логическая формула, которая принимает значение $T_{P}$ всякий раз, когда предметная область находится в определенном состоянии.

Итак, каждая целевая установка сетевой модели характеризуется истинностями: деонтической $\left(T_{D}\right)$ - необходимость достижения целевой установки; алетической $\left(T_{A}\right)$ - возможность достижения целевой установки; прагматической $\left(T_{P}\right)$ - факт достижения целевой установки.

Соотношения значений истинности вершины сетевой модели, адекватно отражающей закономерности предметной области, можно описать двумя аксиомами

$$
\begin{aligned}
& H M \uparrow y \equiv N_{D} y, \\
& H \stackrel{n}{\Lambda} \varphi_{i}(\alpha, t) \equiv N_{D} y,
\end{aligned}
$$

где $H$ - оператор необходимости:

$$
(H a \equiv b) \equiv_{D f} \sim b \rightarrow \sim a .
$$

Оператор достаточности Д, соответственно:

$$
(\text { Д } a \equiv b) \equiv_{D f} b \rightarrow a .
$$

Таким образом, цели, представленные в рамках единого формализма, позволяют формализовать задачи принятия решений в энергетике. С их помощью устанавливается однозначное соответствие между актуализацией целевых установок и фактом их достижения в объективной реальности. 
Для деонтического аспекта знаний, в отличие от алетического, вершина типа "И" имеет двойственность содержания (рис. 1).

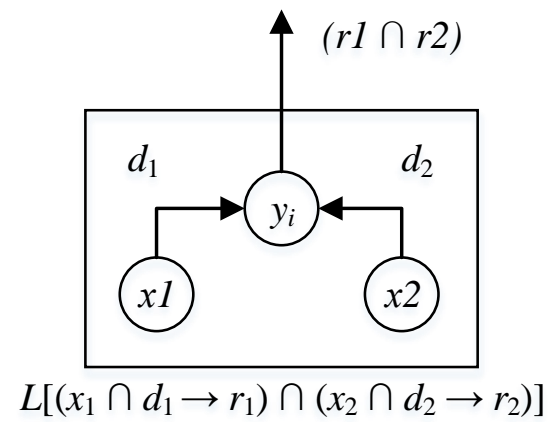

a)

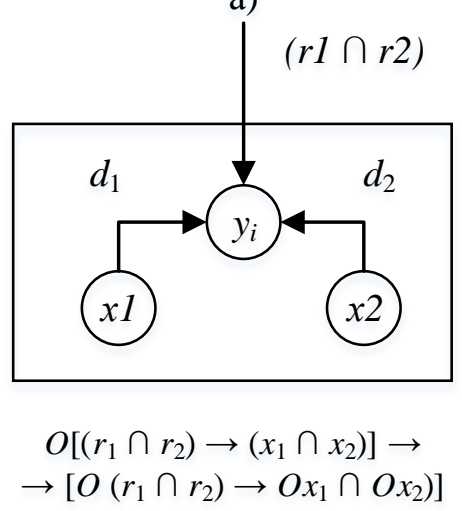

б)

а) алетический аспект, б) деонтический аспект.

Рис. 1. Двойственность для вершины типа 'И' a) for the aletic aspect, b) for the deontic aspect.

Fig.1. Duality for a vertex of type "AND".

Здесь $x_{1}$ и $x_{2}-$ подцели; $d_{1}$ и $d_{2}-$ действия; $r_{1}$ и $r_{2}$ - результаты действий; $L$ и $O$ - операторы алетической и деонтической логики соответственно.

Итак, установлено однозначное соответствие значений истинности разных аспектов знаний и соответствие выражений, описывающих взаимозависимость вершин в системах алетической и деонтической логик.

Для задания формальной системы, кроме выделения множества целей, необходимо задать множество отношений для описания закономерностей функционирования предметной области. Система отношений требует ответа на вопрос о ее "строгости".

Очевидно, для открытой экспертной системы базовая система отношений не обязана быть полной. Но тогда не может быть гарантирована строгость системы отношений. И результатом может стать неразрешимость задачи контроля корректности знаний. Корректное описание закономерностей предметной области достигается представлением каждого отношения отношением строгого порядка [12].

Для моделирования предметной области использованы отношения подчинения, предшествования и типа "начальное условие - результат".

Пусть цель, выраженная формулой $x$, подчинена цели $y$. Цель $x$ является конъюнктивной или дизъюнктивной составляющей $y$ или необходимым условием ее достижения. Тогда отношение подчинения формально описывается как:

$$
\begin{aligned}
& (x \subset y) \equiv_{D f}\left(x \in \Phi_{y}\right) \vee\left(\sim x \in \Phi_{y}\right) \vee \\
& \vee(\sim x \wedge \sim y \rightarrow \sim M \uparrow y),
\end{aligned}
$$

где $\Phi_{y}-$ множество составляющих цели $y$;

$M \uparrow y$ - оператор "возможно в будущем" алетической логики; последний дизъюнктивный член выражает необходимость $x$ для достижения $y$.

Отношение подчинения означает некий порядок целей. Доказано, что отношение подчинения является отношением строгого порядка, т. к. оно антирефлексивно, антисимметрично и транзитивно [13].

Последовательность достижения целей определяет отношения между ними. Пусть цель, описываемая высказыванием $x$, предшествует цели $y$ (обозначается $x T y$ ). Пусть существуют ситуация $\alpha$ и момент времени $t$. Если в этой ситуации и в этот момент времени $x$ - истинно, а $y-$ ошибочно, то имеем отношение слабого предшествования [14]:

$$
\begin{aligned}
& x T y \equiv{ }_{D f} \exists \alpha_{1} \exists t_{1} \exists \alpha_{2} \exists t_{2} x\left(\alpha, t_{1}\right) \wedge \\
& \wedge \sim y\left(\alpha_{1}, t_{1}\right) \wedge y\left(\alpha_{2}, t_{2}\right) \wedge\left(t_{2}>t_{1}\right),
\end{aligned}
$$

где $x(\alpha, t), y(\alpha, t)$ - значение истинности формул $x, y$ в ситуации $\alpha$ в момент $t$.

Фактически отношения слабого предшествования означают установление факта достижения целей.

Отношение предшествования также может быть отношением строгого порядка.

Отношения типа "начальные условия результат" характеризуют возможные действия системы для ее перехода из одного состояния в другое. Для реализации данного 
типа отношений требуется определенный ресурс $W$, характеризуемый своей системой ограничений.

При описании закономерностей функционирования объектов энергетики не всегда возможно выработать однозначный набор действий для перехода из одного состояния в другое. Вершины "разветвители" позволяют представить альтернативные варианты действий. Сплошные дуги отражают действия системы, пунктирные отношения подчинения и штрихпунктирные отношения предшествования.

Корректность сетевых моделей, описывающих задачи в представленной формальной системе, проверяется определением истинности и контролем факта использования целевой установки. При этом каждое из рассмотренных отношений фактически позволяет реализовать процедуру проверки истинности.

Определим более общие характеристики отношения следования.

Целевые установки, связанные с помощью введенных отношений, разнесены по времени.

Отношение следования, связывающее цели $x$ и $y$, в явном виде отражает этот факт $x \rightarrow y$. Для формального описания динамических свойств предметной области для каждого аспекта знаний и временной последовательности достижения промежуточных целей определим свойства данного отношения [14]:

$$
\begin{gathered}
(x \rightarrow y) \rightarrow(a \circ x \rightarrow a \circ y) ; \\
(x \rightarrow y) \rightarrow(x \circ a \rightarrow y \circ a) ; \\
\sim(x \circ y) \rightarrow \sim x \cup \sim y \cup(x \cap y) \cup(y \circ x) ; \\
(x \cup y) \circ z \rightarrow(x \circ z) \cup(y \circ z) ; \\
(x \cap y) \circ z \rightarrow(x \circ z) \cap(y \circ z) ; \\
z \circ(x \cup y) \rightarrow(z \circ x) \cup(z \circ y) ; \\
z \circ(x \cap y) \rightarrow(z \circ x) \cap(z \circ y)
\end{gathered}
$$

Семантическая интерпретация следования между целевыми установками для алетического аспекта знаний отражает следование результата за действием; для деонтического - соотношение необходимых и достаточных условий; для прагматического аспекта знаний - логическое следование, имеющее место в предметной области.

Рассмотрим отношение следования для прагматического аспекта знаний.
Характеристическая функция вида (9), отражающая факт достижения целевой установки, представляется в виде:

$$
x=\bigcup_{i=1}^{I} \bigcap_{j=1}^{n_{i}} \varphi_{i j}
$$

где $\varphi_{i j}$ - элементарная логическая формула, выражающая определенное состояние рассматриваемой области.

В общем случае, множество признаков их можно разделить на доступные для наблюдения системой признаки $-\pi_{i j}$ недоступные $-\xi_{i j}$. Тогда для $x$ :

$$
x=\bigcup_{i=1}^{I}\left(\bigcap_{j=l}^{k_{i}} \pi_{i j}\right) \cap\left(\bigcap_{r=l}^{m_{i}} \xi_{i r}\right),
$$

где $k_{i}+m_{i}=n_{i}$ для всех $i$.

Сетевая модель фактически определяет отношение между признаками, по которым фиксируется достижение целей.

При рассмотрении операции следования $x \rightarrow y$ в рамках прагматичного аспекта знаний необходимо однозначно определить соответствующие ограничения. Так, зафиксировать факт достижения цели $x$ возможно только по совокупности соответствующих $\pi_{i j}$. Однако для $y$ набор фиксирующих признаков может выглядеть как в выражении (24). Это исключает возможность установления факта достижения цели $y$. Т. е., операция следования для сетевой модели может быть строго описана с помощью аппарата топологической булевой алгебры. Для этого введем понятия внутренности $I x$ и замыкания $C x$ и определим их свойства [13]:

$$
\begin{gathered}
(I x \cap I y) \rightarrow I(x \cap y) ; \\
I x \rightarrow x ; \\
I x \rightarrow I I x ; \\
I(x \cup \sim x) .
\end{gathered}
$$

Операция замыкания определяется как:

$$
C x \equiv \sim I \sim x .
$$

Выразим внутренность и замыкание через совокупности наблюдаемых и ненаблюдаемых признаков: 


$$
\begin{aligned}
& I x=\bigcup_{i=1}^{I}\left(\bigcap_{j=1}^{k_{i}} \pi_{i j}\right) ; \\
& C x=\bigcup_{i=1}^{I}\left(\bigcap_{j=1}^{k_{i}} \pi_{i j}\right) \bigcap\left(\bigcap_{r=1}^{m_{i}} \xi_{i r}\right) .
\end{aligned}
$$

Учитывая, что

$$
C \sim x \equiv \sim I x, \quad I \sim x \equiv \sim C x,
$$

образуем вектор в виде кортежа формул:

$$
\bar{x}=\langle I x, \quad C \sim x, \quad C x, \quad I \sim x\rangle,
$$

обеспечивающий однозначное установление факта достижения цели $x$.

Если интерпретировать $I$ как оператор необходимости $L$, а $C$ - как оператор возможности $M$ и определить элементы вектора $A \Rightarrow B$, то получаем:

$$
\begin{aligned}
& \overline{(A \Rightarrow B)} \equiv\left\langle\begin{array}{l}
I(A \Rightarrow B), C \sim(A \Rightarrow B), \\
C(A \Rightarrow B), I \sim(A \Rightarrow B)
\end{array}\right\rangle ; \\
& \overline{(A \Rightarrow B)} \equiv\left\langle\begin{array}{l}
L(A \Rightarrow B), M(A \cap \sim B), \\
M(A \Rightarrow B), L(A \cap \sim B)
\end{array}\right\rangle
\end{aligned}
$$

Проводим замену:

$$
L \sim A \cup L B \Rightarrow L \sim A \cup B,
$$

которую заменим эквивалентной формулой:

$$
(M A \Rightarrow L B) \Rightarrow L(A \Rightarrow B),(37)
$$

и раскроем импликацию:

$$
\begin{aligned}
M(A \Rightarrow B) \equiv M \sim A \cup M B, & \\
& M \sim A \cup M B \equiv L A \Rightarrow M B .
\end{aligned}
$$

Распространим принцип (36) на $M(A \cap B)$. Перепишем выражение (36) в эквивалентной форме:

$$
\sim M \sim(\sim A \cup \sim B) \cup \sim L \sim A \bigcap \sim L \sim B, \quad(40)
$$

что соответствует выражению:

$$
M(A \cap B) \Rightarrow M A \cap M B .
$$

В результате получаем следующий вектор:

$$
\overline{(A \Rightarrow B)} \equiv\left\langle\begin{array}{l}
M A \Rightarrow L B, M A \cap M \sim B, \\
L A \Rightarrow M B, L A \cap \sim L B
\end{array}\right\rangle
$$

Проанализируем элементы этого вектора.

Согласно принятой интерпретации, первый элемент вектора (42) обуславливает:

а третий элемент (42):

$$
C A \subset I B,
$$

$$
I A \subset C B .
$$

Таким образом, для рассматриваемой операции следования имеет место:

$$
(C A \subset I B) \cap(I A \subset C B) .
$$

Операция следования, соответствующая (45), определяет несколько иную строгость следования по сравнению с классическим следованием, для которого:

$$
(I A \subset I B) \cap(C A \subset C B) .
$$

Таким образом, выражение (46) означает, если $A \rightarrow B$, то наблюдаемые признаки целевой установки $A$ из $C A$ могут определять наличие наблюдаемых признаков для $B$, то есть $I B$, но не наоборот. Следовательно, операция присоединения следствий исследуемой формальной системы позволяет контролировать корректность эквивалентных преобразований формализованных описаний.

Рассмотрим классификацию задач принятия решений в предложенных терминах системы понятий и отношений. Рассмотрим дизъюнктивные задачи выбора решений (рис. 2).

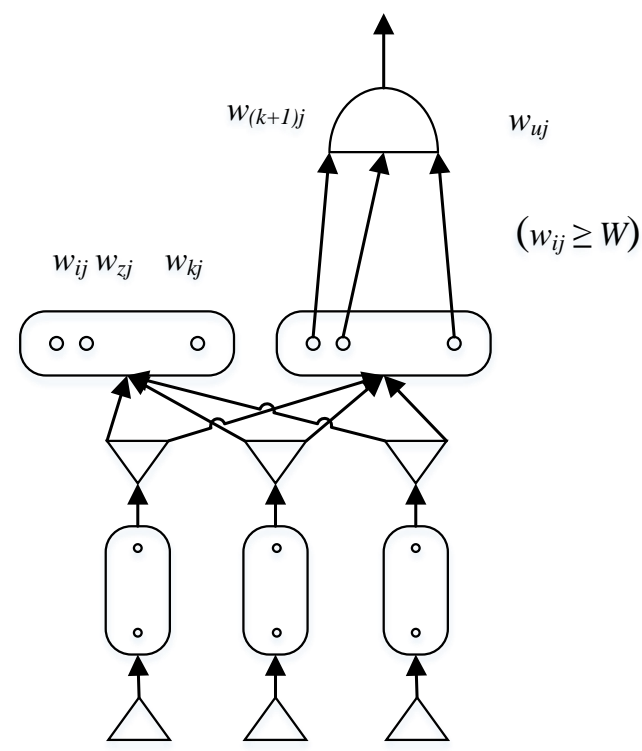

Рис. 2. Дизъюнктивная задача выбора решений.

Fig. 2 - Disjunctive decision problem.

В первую очередь задачи принятия решений возникают в дизъюнктивных вершинах сети. В них есть несколько вариантов достижения цели. Необходимо из них выбрать наилучший в некотором смысле (обеспечить $\max W$ или приемлемый 
$\left(w_{i j}>\bar{w}\right)$, где $\bar{w}-$ некоторое пороговое значение функции полезности результата, а $w_{i j}$ - значение функции полезности рассматриваемого $j$-го варианта $i$-ой дизъюнктивной вершины) [15]. Результаты принимаемых решений могут быть неопределенными либо случайными для системы. Причем часть альтернативных результатов может быть отнесена к благоприятным (приемлемым), а часть - к неблагоприятным.

Второй тип задач соответствует конъюнктивной вершине сети. Он возникает, когда для достижения каждой из подчиненных конъюнктивных составляющих необходиы определенные затраты некоторого ресурса $r_{i j}$. Параметры использования ресурса определяют возможность достижения цели по каждой из конъюнктивных составляющих. Пусть суммарный запас ресурса ограничен. Тогда необходимо распределить его между составляющими (ветвями модели) так, чтобы обеспечить достижение всех конъюнктивных составляющих в приемлемое время. При наличии несколько типов ресурсов, необходимых для достижения каждой из составляющих, возникает - задача распределения ресурса (рис. 3).

Пусть на сетевой модели для планирования процесса достижения цели необходимо принять несколько решений (многошаговые задачи). Каждое из них соответствует множеству вершин сети, упорядоченных по одному из отношений подчинения, предшествования, действия. Тогда каждый из вариантов решений на каждой из вершин сети не может рассматриваться обособленно (рис. 4).

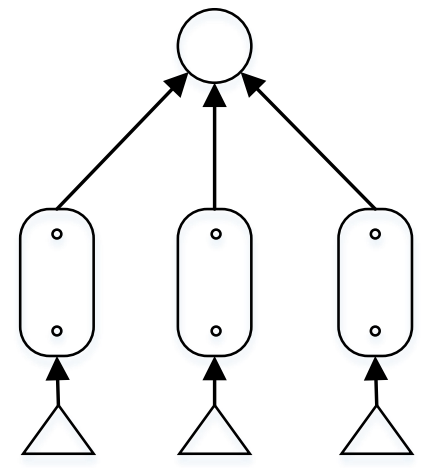

Рис. 3. Конъюнктивная задача выбора решений.

Fig. 3. Conjunctive decision problem
Описание динамических свойств предметной области

Множество требований, предъявляемых к аппарату формализации параметров динамической системы, сформулировал Эшби [16] для исследования адаптивного поведения. Строгое описание сетевой модели возможно в рамках алетической и деонтической логик и представления прагматических знаний. Расширение возможностей алетической и деонтической логик системой модально-временных операторов нашло применение для решения целого ряда практических задач [12-16], но не определено формально.

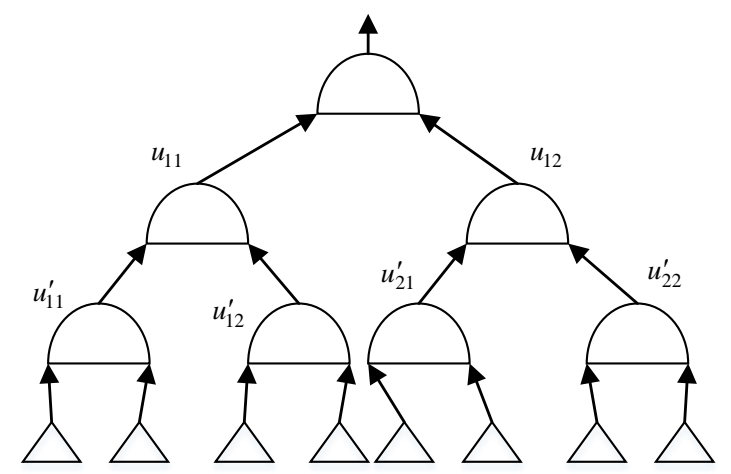

Рис. 4. Многомерное представление решения задачи принятия решений.

Fig. 4. Multidimensional representation of the decision problem solution.

Логическому выводу в рассматриваемых динамических теориях предшествует распознавание применимости их аксиом при данном состоянии реальности. Для этого используются исходные условия сетевой модели, имеющие значение "истина" для относительно узкого диапазона свойств. В этом случае динамическая теория представляется в виде системы аксиом $a_{i} \rightarrow \Phi_{i}(i=1,2, \ldots, N)$, где $a_{i}$ - формула, соответствующая $i$-му начальному условию; $\Phi_{i}$ - формула, соответствующая целевой установке.

Пусть существует некоторая формулавопрос $Q$, ответ на который должен быть получен с использованием динамической теории. Тогда строится доказательство выводимости:

$$
\Phi_{i_{1}}^{\prime}, \Phi_{i_{2}}^{\prime}, \ldots, \Phi_{i_{k}}^{\prime} \mapsto Q
$$

в котором отображаются все возможные варианты вывода $Q$ с $\Phi_{i_{s}}^{\prime}$, где $\Phi_{i_{s}}^{\prime}-$ это $L \Phi_{i_{s}}$ или $M \Phi_{i_{s}}$. Каждому из указанных вариантов 
доказательства соответствует конъюнкция $\alpha_{i_{1}}^{\prime} \wedge \alpha_{i_{2}}^{\prime} \wedge \ldots \wedge \alpha_{i_{k}}^{\prime}$. Здесь $\alpha_{i_{s}}^{\prime}-$ это $L \alpha_{i_{s}}$, если $\Phi_{i_{s}}^{\prime}=L \Phi_{i_{s}}, \quad$ и $\quad \alpha_{i_{s}}^{\prime}=M \alpha_{i_{s}}$, если $\Phi_{i_{s}}^{\prime}=M \Phi_{i_{s}}$. Таким образом, динамическая теория позволяет получать программы поиска ответов на вопросы. Для этого используются реальные данные и локализованное множество аксиом формальной теории.

С точки зрения лиц, принимающих решения, модель динамики системы должна представляться в виде конечного множества классов эквивалентных состояний. Состояния описываются формулами, составленными из признаков. Каждый такой класс эквивалентности может иметь собственную структуру. Она детализирует описание со степенью подробности, достаточной для реализации управления.

Таким образом, для реализации возможных вариантов действий в системах реального времени необходимо установить факт достижения каждой целевой установки. То есть определяются значения истинности функции (23), элементы $\varphi_{i j}$ которой принимают двоичные значения и составляют в общем случае вектор $\vec{\Phi}$.

Описание динамических свойств системы в рамках прагматичного аспекта знаний

Пусть $\quad \vec{X} \quad$ и $\vec{Z}$ - два вектора вида $\dot{\Phi}=<\varphi_{i j}>, \quad i=1,2, \ldots, I ; \quad j=1,2, \ldots, n_{i} \quad$ из двоичного пространства $B^{k}$. Тогда существует единственный вектор $\vec{y} \in B^{k}$, такой, что $\vec{x} \oplus \vec{y}=\vec{z}$.

Вектор $\vec{x} \oplus \vec{y}=\vec{z}$ называется разницей векторов $\vec{z}$ и $\vec{x}$ и может быть рассчитан с помощью сложения по модулю 2 [17]. Рассмотрим $i$-ые составляющие $x_{i}, y_{i}, z_{i}$, $i=1,2, \ldots, n$ :

a) $y_{i}=0$, если $x_{i}=z_{i}$ как при $x_{i}=z_{i}=0$, так и при $x_{i}=z_{i}=1$;

б) $y_{i}=1$ при $x_{i} \neq z_{i}$, то есть для $x_{i}=0, y_{i}=1$ и для $x_{i}=1, y_{i}=0$.

Значения компоненты $y_{i}$ являются таким образом двоичной мерой изменения компоненты $x_{i}$ на пути от точки $\vec{X}$ до точки $\vec{Z}$. Если $x_{i}$ является постоянным, то $y_{i}=0$. Равенство $y_{i}=1$ свидетельствует об изменении значения $x_{i}$ (рис. 5).

\begin{tabular}{|c|c|c|}
\hline$x_{1} \ldots x_{i} \ldots x_{k}$ & $x_{1} \ldots x_{i} \ldots x_{k}$ & $y_{i}$ \\
\hline 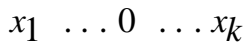 & $\begin{array}{lllll}x_{1} & \ldots & 0 & \ldots & x_{k}\end{array}$ & 0 \\
\hline $\begin{array}{lllll}x_{1} & \ldots & \ldots & \ldots & x_{k}\end{array}$ & $x_{1} \ldots \begin{array}{lll}\ldots & \ldots & x_{k}\end{array}$ & 1 \\
\hline$x_{1} \ldots 1 \ldots x_{k}$ & $\begin{array}{lllll}x_{1} & \ldots & 0 & \ldots & x_{k}\end{array}$ & 1 \\
\hline$x_{1} \ldots 1 \ldots x_{k}$ & $x_{1} \ldots 1 \ldots x_{k}$ & 0 \\
\hline
\end{tabular}

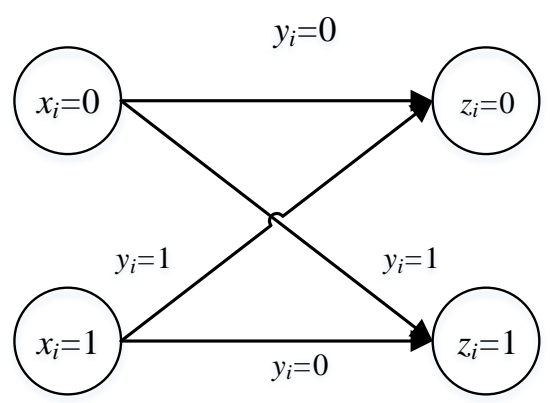

Рис. 5. Характеристика вектора $\vec{y}$ в простанстве $B^{k}$.

Fig. 5. Characteristic of vector $\vec{y}$ in space $B^{k}$.

Чем больше единиц в векторе $\vec{y}$, тем больше компонентов $\vec{x}$ должны менять свое значение при переходе от $\vec{X}$ к $\vec{Z}$.

На рис. 6 показаны соответствующие разницы $B^{3}$.

Итак, в пространстве $B^{k}$ рассмотрены две точки $\vec{X}$ и $\vec{Z}$ и определено, что изменения при переходе от $\vec{X}$ до $\vec{Z}$ могут быть описаны с помощью разницы $\vec{y}=\vec{x} \oplus \vec{z}=\vec{z} \oplus \vec{x}$. Рассмотрим, что происходит при изменении одной компоненты $x_{i}$ и при ее постоянстве.

Опишем процесс изменения значений истинности $\Delta \varphi_{i}\left(\alpha, \beta, t_{1}, t_{2}\right)$ элементарных формул $\varphi_{i}(\alpha, t)$, входящих в состав характеристической функции (9).

Процесс изменения значений истинности элементарных формул представим, введя переменную $d \varphi_{i}$ с такими значениями [13]:

$d \varphi_{i}=\left\{\begin{array}{l}1, \text { если элементарная формула меняет } \\ \text { своё значение; } \\ 0, \text { если элементарная формула не меняет } \\ \text { своё значение. }\end{array}\right.$

Таким образом, речь идет о двоичной переменной $d \varphi_{i}$, которая описывает факт 
изменения значений истинности $\varphi_{i}$.

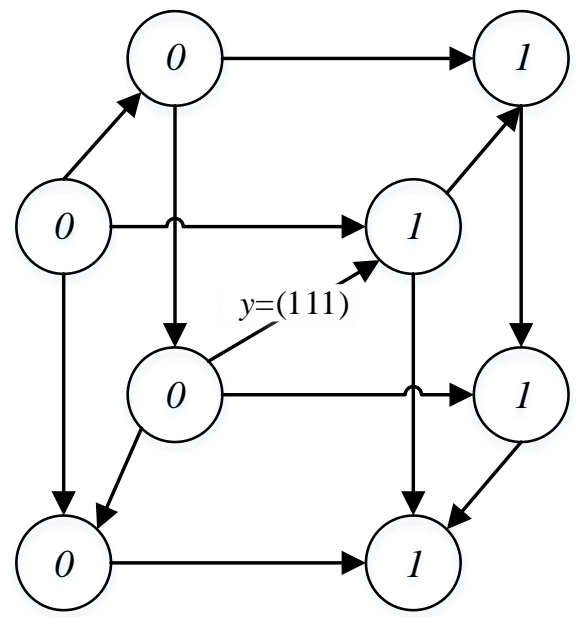

Рис. 6. Характеристика вектора $\vec{y}$ в простанстве $B^{3}$.

Fig. 6. Characteristic of vector $\vec{y}$ in space $B^{3}$.

Так, четыре возможные ситуации (рис. 7), выражаются соотношением $\kappa_{i} \oplus d \varphi_{i}=\varphi_{i}{ }^{*}$, где $\varphi_{i}^{*}$ - значения элементарной формулы при ситуации $\beta$. Однако из рис. 7 не видно, как изменяются значения истинности элементарных формул. Так, для случаев а) и б) (рис. 7) $d \varphi_{i}=1, \quad$ хотя предыдущее состояние для случая а) имеет значение 1 , а для случая б) -0 .

Рассмотрим факт достижения определенной целевой установки $A$ по наблюдаемым признакам. Пусть значение истинности соответствующего признака однозначно связано со значениями истинности признаков целевой установки $B$, при $A \rightarrow B$. Тогда подобное описание динамических свойств является неприемлемым.

Определим изменение значений истинности формул для предыдущих состояний [15]:

$$
\begin{aligned}
& \Delta \varphi_{i}\left(\alpha, \beta, t_{1}, t_{2}\right) \equiv_{D f} \varphi_{i}\left(\alpha, t_{1}\right) \wedge \\
& \wedge \sim \varphi_{i}\left(\beta, t_{2}\right) \vee \sim \varphi_{i}\left(\alpha, t_{1}\right) \wedge \varphi_{i}\left(\beta, t_{2}\right),
\end{aligned}
$$

где

\section{выражает}

$$
\Delta^{+}\left(\alpha, \beta, t_{1}, t_{2}\right) \equiv \sim \varphi_{i}\left(\alpha, t_{1}\right) \wedge \varphi_{i}\left(\beta, t_{2}\right)
$$
$\Delta^{-}\left(\alpha, \beta, t_{1}, t_{2}\right) \equiv \varphi_{i}\left(\alpha, t_{1}\right) \wedge \sim \varphi_{i}\left(\beta, t_{2}\right) \quad$ выражает отрицательное изменение (чувствительно к случаю б) рис. 7).

Фактически $\Delta \varphi_{i}\left(\alpha, \beta, t_{1}, t_{2}\right) \quad$ описывает изменения значения истинности $\varphi_{i}$ на конечных интервалах времени.

Из определения (47) следуют правила расчета изменений для дизъюнкции и конъюнкции формул.

$$
\begin{aligned}
& \text { Если } \\
& \varphi(\alpha, t) \equiv \bigvee_{k=1}^{m} \varphi_{k}(\alpha, t), \\
& \Delta \varphi_{i}\left(\alpha, \beta, t_{1}, t_{2}\right) \equiv\left(\bigvee_{k=1}^{m} \varphi_{k}(\alpha, t)\right) \bigwedge_{k=1}^{m}\left(\sim \varphi_{k}\left(\alpha, t_{1}\right) \wedge\right. \\
& \wedge \sim \Delta \varphi_{k}\left(\alpha, \beta, t_{1}, t_{2}\right) \vee \varphi_{k}\left(\alpha, t_{1}\right) \wedge \\
& \left.\wedge \varphi_{k}\left(\alpha, \beta, t_{1}, t_{2}\right)\right) \vee \sim\left(\bigvee_{k=1}^{m} \varphi_{k}\left(\alpha, t_{1}\right)\right) \wedge \\
& \wedge\left(\bigvee_{k=1}^{m} \Delta \varphi_{k}\left(\alpha, \beta, t_{1}, t_{2}\right)\right)
\end{aligned}
$$

Если $\varphi(\alpha, t) \equiv \bigwedge_{k=1}^{m} \varphi_{k}(\alpha, t), \quad$ то

$$
\Delta \varphi_{i}\left(\alpha, \beta, t_{1}, t_{2}\right) \equiv\left(\bigwedge_{k=1}^{m} \varphi_{k}(\alpha, t)\right) \wedge\left(\bigvee_{k=1}^{m} \Delta \varphi_{k}\left(\alpha, \beta, t_{1}, t_{2}\right)\right) \vee
$$
$\alpha, t_{1}$
$\beta, t_{2}$
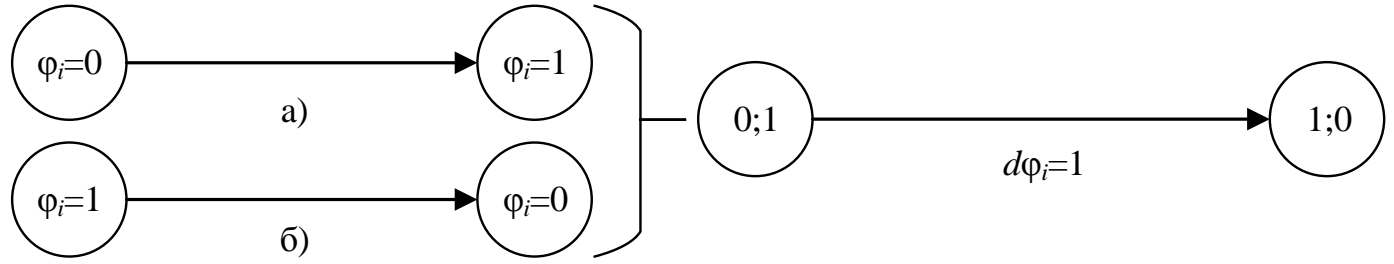

$d \varphi_{i}=1$

$\varphi_{i}=0$

б)
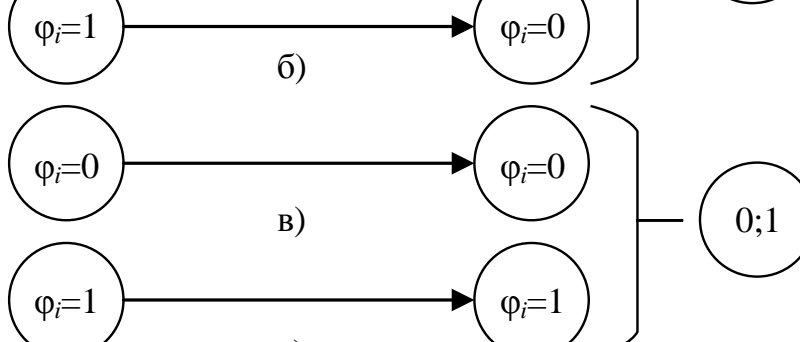

в)
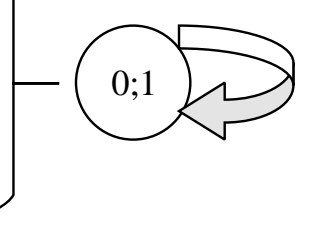

$d \varphi_{i}=0$

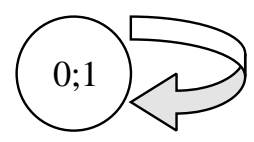

г)

Рис. 7. Переход из ситуации $\alpha$ к ситуации $\beta$.

Fig. 7. Transition from situation $\alpha$ to situation $\beta$. 


$$
\begin{aligned}
& \vee \sim\left(\bigwedge_{k=1}^{m} \varphi_{k}(\alpha, t)\right) \bigwedge_{k=1}^{m}\left(\sim \phi_{k}(\alpha, t) \wedge \Delta \varphi_{k}\left(\alpha, \beta, t_{1}, t_{2}\right)\right) \vee \\
& \left.\vee \varphi_{k}(\alpha, t) \wedge \sim \Delta \varphi_{k}\left(\alpha, \beta, t_{1}, t_{2}\right)\right) .
\end{aligned}
$$

Таким образом, полученный формальный аппарат позволяет локализовать начальную систему аксиом, представленных в сетевой модели, проверять выводимость формулвопросов и устанавливать однозначное соответствие между наблюдаемыми признаками ситуации в предметной области и фактом достижения целевой установки.

При синтезе взаимосвязанных структур целевых установок, отражающих свойства предметной области, может возникнуть такой случай. Пусть значения характеристических функций вида $\varphi(\alpha, t)$, определяющих факт достижения конечного множества целей, не зависят от $\alpha$ и $t$. Фактически данные цели описывают желаемые состояния объектов среды и системы. Для этого указываются их необходимые свойства или отношения. Такая цель постоянна и распространяется на все ситуации в среде $(\forall \alpha)$, на все моменты времени $(\forall t)$ и на все объекты, рассматриваемые в рамках решаемой задачи.

Цели, достижение которых зависит от ситуации, называются оперативными. Фактически оперативная цель является интерпретацией постоянной цели. Она определяет актуальность, возможность и установление факта достижения для конкретных объектов и свойств, имеющих место для текущих $\alpha$ и $t$. Если некоторое множество объектов $O_{1}, O_{2}, \ldots, O_{k}$, имеют такие признаки, то формулируется $k$ оперативных совместно достижимых целей. В общем случае, для достижения конкретной оперативной цели необходимо достижение некоторого множества промежуточных целей, связанных между собой отношениями.

Зная структуру оперативных целей одной из противодействующих систем $S_{1}$, можно получить соответствующую структуру целей другой системы $S_{2}$.

В общем случае выражение для оперативной цели системы выглядит так:

$$
O\left[a_{i} \wedge\left(L_{t} \uparrow h_{i}\right)\right],
$$

где $O$ - один из модальных временных операторов; $h_{i}$ - вспомогательный предикат, принимающий значение "истина" при $t>t_{\text {доn }}$. Здесь $t_{\text {до }}$ - момент времени, к которому указанная цель имеет место (актуальна).

Используя правила интерпретации связанных модально временных операторов

$$
\begin{aligned}
& L \uparrow P \equiv_{D f} \sim M_{t} \uparrow \sim P, \\
& \sim L \uparrow P \equiv_{D f} M_{t} \uparrow \sim P,
\end{aligned}
$$

можно получить выражение для цели, достижение которой нарушает необходимые условия достижения цели $a_{1}$ системы $S_{2}$ :

$$
O^{*}\left[\sim a_{i} \vee M_{t} \downarrow h_{i}\right],
$$

где $O^{*}$ - временной оператор, связанный с $O$, образованный по этому правилу.

Пусть выражение для оперативной цели системы $S_{2}$ имеет вид $M_{t} \uparrow\left[a_{i} \wedge\left(L_{t} \downarrow \sim h_{i}\right)\right]$ и $a_{1} \wedge a_{2} \wedge \ldots \wedge a_{m}$. Тогда составная цель системы $S_{1}$ при условии $b_{i}=\sim a_{i}$ имеет вид $L_{t} \uparrow\left[\sim b_{1} \vee \sim b_{2} \vee \ldots \vee \sim b_{m} \vee M_{t} \downarrow h_{i}\right] . \quad$ Это объясняется тем, что операторы $M_{t} \uparrow$ и $L_{t} \uparrow$ симметричны относительно оси $D$ (рис. 8). Для недостижения системой $S_{2}$ конечной конъюнктивной цели достаточно воспрепятствовать достижению одной из ее составляющих.

Дизъюнктивной цели систем $S_{2}$ будет отвечать цель $S_{1}$ в виде конъюнкции ее составляющих, взятых со знаком отрицания.

Используя свойство временных операторов

$$
L_{t} \uparrow p \vee L_{t} \uparrow q \rightarrow L_{t} \uparrow(p \vee q),
$$

заменим данную целевую установку на цель:

$$
b_{i}^{*}=L_{t} \uparrow\left(\sim b_{1} \vee \sim b_{2} \vee \ldots \vee \sim b_{m}\right),
$$

Дополнительно укажем, что цель должна выполняться вплоть до момента времени, когда $h_{i}$ примет значение “истина $D$ ”.

Аналогично строятся выражения и для дизъюнктивной цели. Если для системы $S_{2}$ $c_{i}=c_{1} \vee c_{2} \vee \ldots \vee c_{m}$, то соответствующая цель $\begin{array}{lcl}\text { системы } & S_{2} & d_{i}=\sim c_{i} \\ d_{i}=L_{t} \uparrow\left[\left(\sim d_{1} \wedge \sim d_{2} \wedge \ldots \wedge \sim d_{m}\right) \vee M_{t} \downarrow h_{i}\right] .\end{array}$ 
Используя свойство операторов (50), цели, связанной с отношениями

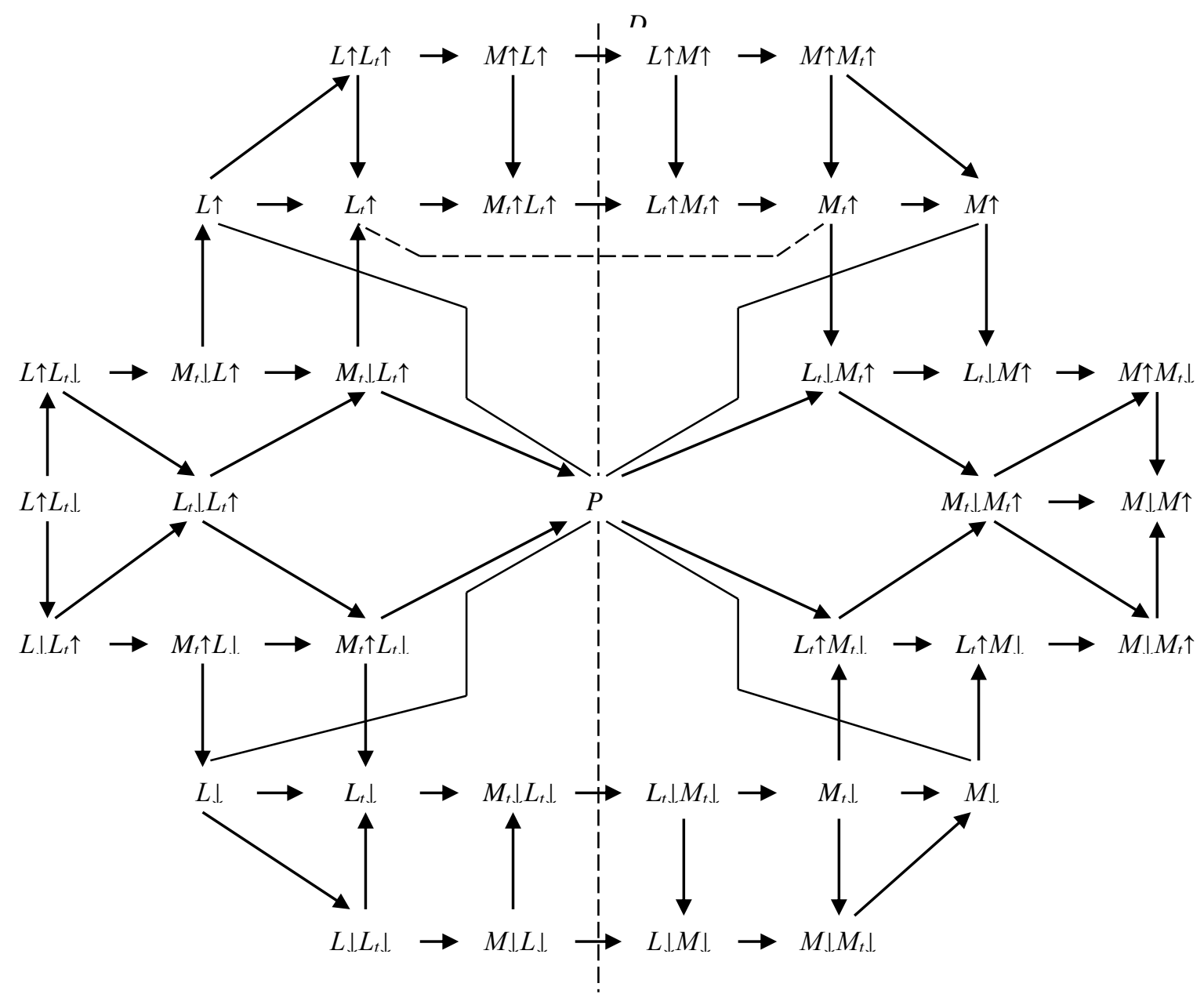

Рис. 8. Симметричность временных операторов.

Fig. 8. Symmetry of temporary operators.

получаем целевую установку $d_{i}^{*}=L_{t} \uparrow\left[\sim d_{1} \wedge \sim d_{2} \wedge \ldots \wedge \sim d_{m}\right] \quad$ с указанием, как и для цели (50).

Рекурсивный характер рассмотренных правил для конъюнктивных составляющих примет следующий вид:

$$
\begin{aligned}
& \forall t_{1} \sim b_{1}\left(t_{1}\right) \wedge \forall t_{2} \sim b_{2}\left(t_{2}\right) \leftrightarrow \\
& \leftrightarrow \forall t\left[\sim b_{1}\left(t_{1}\right) \wedge \sim b_{2}\left(t_{2}\right)\right]
\end{aligned}
$$

Можно принять тот же принцип, когда $b_{1}$ и $b_{2}$ имеют конечную продолжительность во времени. В этом случае условие ненаступления цели $a$ имеет вид:

$$
\begin{aligned}
& \sim a \equiv L_{t} \uparrow\left(\sim b_{1} \wedge b_{2} \vee b_{1} \wedge \sim b_{2}\right) \vee \\
& \vee L_{t} \uparrow \sim b_{1} \vee L_{t} \uparrow \sim b_{2} .
\end{aligned}
$$

Таким образом, условиями недостижения предшествования, являются:

a) изменение последовательности наступления предыдущих событий;

б) ненаступление одного или нескольких предыдущих событий;

в) совместимость во времени наступления начал событий $a$ i $b$ (последний дизъюнктивный член).

Графическая интерпретация указанного правила представлена на рис. 9. Оно разрешает получить структуру целей системы $S_{1}$ из структуры целей системы $S_{2}$, связанных отношениями предшествования. Цели системы $S_{1}$ являются конъюнктивными и дизъюнктивными составляющими предшествующих целей в структуре целей системы $S_{2}$. Т. е. при преобразовании сетевой модели $S_{2}$ в структуре целей $S_{1}$ отношение предшествования между целями системы $S_{2}$ изменяется на отношение подчинения. 


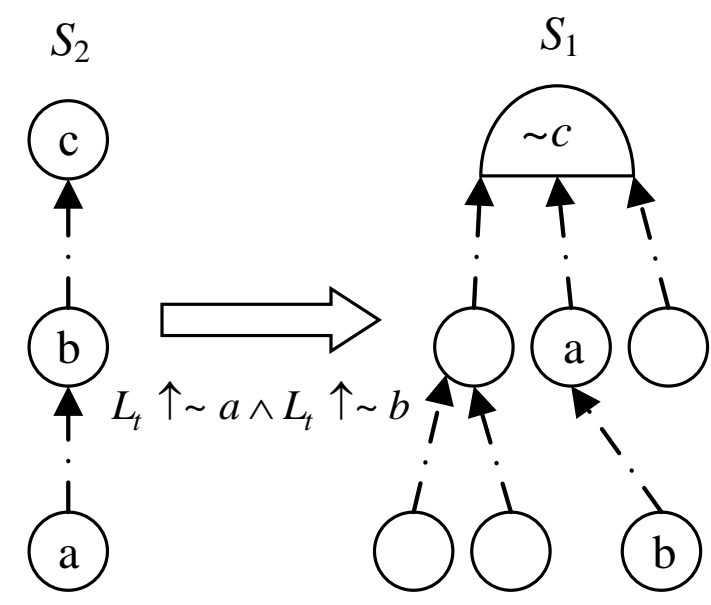

Рис. 9. Структуры целей систем $S_{2}$, и $S_{1}$, связанных отношениями предшествования и подчинения.

Fig. 9. Structures of the goals of systems 2 and 3 , connected by relations of precedence and subordination.

\section{ВЫВОДЫ}

Таким образом, основу структуры аппарата формализации задач принятия решений для систем управления реального времени составляют целевые установки, описываемые в рамках единого формализма. Это позволяет установить однозначное соответствие между процессами актуализации и достижения целевых установок в системе управления и фактом их достижения (недостижения) в объективной реальности.

Множество отношений, определенное на множестве целевых установок, позволяет выражать отношение присоединения следствий при описании закономерностей проблемной области по каждому из аспектов знаний. Сетевая модель, определяя отношение следования между целевыми установками, фактически определяет соответствующее отношение между признаками, по которым в системе управления фиксируется достижение целей. При этом строгость операции присоединения следствий не нарушается.

Введенная система базовых понятий и отношений позволяет классифицировать все множество задач принятия решений. При этом обеспечивается описание динамических свойств системы управления в рамках каждого аспекта знаний.

Разработанный подход к формализации обеспечивает объединение в рамках единого формализма описаний динамических свойств и логико-аналитической деятельности, представленных в разных классах формальных логик, и строгое описание различных аспектов знаний в рамках единого формального аппарата.

Предложенные ситуационные алгоритмы описывают значительный класс аварийных состояний энергосистем. Класс аварийных состояний можно расширить, что потребует построения новых ситуационных алгоритмов.

Ситуационные алгоритмы математически представляют собой систему постулатов некоторой теории. При этом для каждой системы постулатов проверяется, является ли она внутренне непротиворечивой. Это теорема Геделя о неполноте формальной арифметики. Она трактуется как невозможность создания системы поддержки принятия решений, решающей все задачи предметной области, оставаясь в самой области правил. Т. е. параллельнопоследовательное выполнение ситуационных алгоритмов управления может приводить к истинным и ложным утверждениям, т.е. вести к цели и наоборот.

Непротиворечивость теории может быть доказана путем использования постулатов и методов, выходящих за рамки рассматриваемой теории. Ситуационные алгоритмы должны управляться стратегией, которая не может быть внутренним свойством открытой системы, каковой является энергосистема. Стратегия должна представлять собой внешнее содержательное знание не только о целях, но и о налагаемых ограничениях функционирования системы на заданном интервале времени. Цели обычно сохраняют достаточную устойчивость, в то время как ограничения являются варьируемыми величинами, отражающими оптимальную реакцию системы на изменяющиеся внешние условия.

Направлением дальнейших исследований является построение соответствующей формальной теории на основе предложений формальной системы.

\section{Библиография (References)}

[1] Evelio J. González, Leopoldo Acosta Sánchez and Alberto F. Hamilton Castro. Artificial Intelligence Resources in Control and Automation Engineering. DOI: $10.2174 / 97816080512671120101$. 
[2] Spyros G. Tzafestas. Intelligent Systems, Control and Automation: Science and Engineering. January 2016, Intelligent Systems 80.DOI:10.1007/978-3-319-21422-1

[3] Gupta, N.A. Literature Survey on Artificial Intelligence. 2017. Available online: https://www.ijert.org/ research/a-literaturesurvey-on-artificial-intelligence-

IJERTCONV5IS19015.pdf (accessed on 15 December 2020).

[4] Russell, Stuart J. and Peter Norvig. 2020. Artificial Intelligence: A Modern Approach, 4th ed. New York: Pearson.

[5] Marcus, Gary and Ernest Davis. 2020b. Rebooting AI: Building Artificial Intelligence We Can Trust. New York: Penguin

[6] Masnikosa, V.P. The Fundamental Problem of an Artificial Intelligence Realization. Kybernetes? 1998, 27, 71-80.

[7] Metaxiotis, K.; Ergazakis, K.; Samouilidis, E.; Psarras, J. Decision Support Through Knowledge Management: The Role of the Artificial Intelligence. Inf. Manag. Comput. Secur. 2003, 11, 216-221.

[8] Raynor, W.J. The International Dictionary of Artificial Intelligence. Ref. Rev. 2000, 14, 1-380.

[9] Stefanuk, V.L.; Zhozhikashvili, A.V. Productions and Rules in Artificial Intelligence. Kybernetes? 2002, 31, 817-826.

[10] Oke, S.A. A Literature Review on Artificial Intelligence. Int. J. Inf. Manag. Sci. 2008, 19, 535-570.

[11] Carvalho, T.P.; Soares, F.A.A.M.N.; Vita, R.; da Francisco, P.R.; Basto, J.P.; Alcalá, S.G.S. A Systematic Literature Review of Machine Learning Methods Applied to Predictive Maintenance. Comput. Ind. Eng. 2019, 1, 1-12.

[12] Duan, Y.; Edwards, J.S.; Dwivedi, Y.K. Artificial Intelligence for Decision Making in the Era of Big Data-Evolution, Challenges and Research Agenda. Int. J. Inf. Manag. 2019, 48, 63-71.

[13] Stone, P., Brooks, R., Brynjolfsson, E., Calo, R., Etzioni, O., Hager, G., \& Teller, A. (2016, September). One Hundred Year Study on Artificial Intelligence. Artificial Intelligence and Life in 2030. Retrieved from https://ai100.stanford.edu/2016-report

[14] Kareem Ayoub and Kenneth Payne, "Strategy in the Age of Artificial Intelligence," The Journal of Strategic Studies, Vol. 39. No. 5, November 2015, p. 816.

[15] Bughin, J., Hazan, E., Ramaswamy, S., Chui, M., Allas, T., Dahlström, P., ... \& Trench, M. (2017). Artificial Intelligence: The Next Digital Frontier. McKinsey Global Institute, 1-80. Retrieved

from https://www.mckinsey.com/ /media/McKinsey/I ndustries/Advanced\%20Elec

tronics/Our\%20Insights/How\%20artificial\%20int elligence $\% 20$ can $\% 20$ deliver

$\% 20$ real\%20value\%20to\%20companies/MGI-

Artificial-Intelligence Discussion-Paper.ashx

[16] Gofman, M., \& Jin, Z. (2020, October 26). Artificial Intelligence, Education, and Entrepreneurship. Retrieved from http://gofman.info/AI/AI_GofmanJin.pdf

[17] McGovern A., Elmore K. L., Gagne D. J., Haupt S. E., Karstens C. D., Lagerquist R., Williams J. K. Using Artificial Intelligence to Improve Real-Time Decision-Making for HighImpact Weather. Bulletin of the American Meteorological Society, 2017, vol. 98(10), pp. 2073-2090. Doi: 10.1175/BAMS-D-16-0123.1

[18] Chapman C. S., Gallivan J. P., Wood D. K., Milne J. L., Culham J. C., Goodale M. A. Reaching for the Unknown: Multiple Target Encoding and Real-Time Decision-Making in a Rapid Reach Task. Cognition, 2010, vol. 116(2), pp. 168-176. Doi: 10.1016/j.cognition.2010.04.008

[19]Brézillon P. A Contextual Methodology for Modelling Real-Time Decision-Making Support. Supporting Real Time Decision-Making, 2011, vol. 13 , pp. 65-88|.

[20]Decision Support System (DSS). Available at: https://www.investopedia.com/terms/d/decisionsupport-system.asp (accessed 25.10.2021).

[21] Lucarelli S., Marrone A., Niccolò Moro F. NATO Decision-Making in the Age of Big Data and Artificial Intelligence. Brussels - Belgium, 2021. $100 \mathrm{p}$.

[22] Golnaraghi F., Kuo B. Automatic Control Systems. 10th Edition. 2018. 864 p.

[23] Soroudi A., Amraee T. Decision Making under Uncertainty in Energy Systems: State of the Art. Renewable and Sustainable Energy Reviews, 2013, vol. 28, pp. 376-384.

[24] Gonzalez C. Decision Support for Real-Time, Dynamic Decision-Making Tasks. Organizational Behavior and Human Decision Processes, 2005, vol. 96(2), pp. 142-154.

[25]Zhao P., Suryanarayanan S., Simoes M. G. An Energy Management System for Building Structures Using a Multi-Agent Decision-Making Control Methodology. IEEE Transactions on Industry Applications, 2012, vol. 49(1), pp. 322330.

[26] K. Aksyonov, E. Bykov, O. Aksyonova, A. Nevolina, N. Goncharova. Architecture of the multi-agent resource conversion processes extended with agent coalitions. Proceedings of IEEE International Symposium on Robotics and Intelligent Sensors, 221-226, 2016. 
Сведения об авторах.
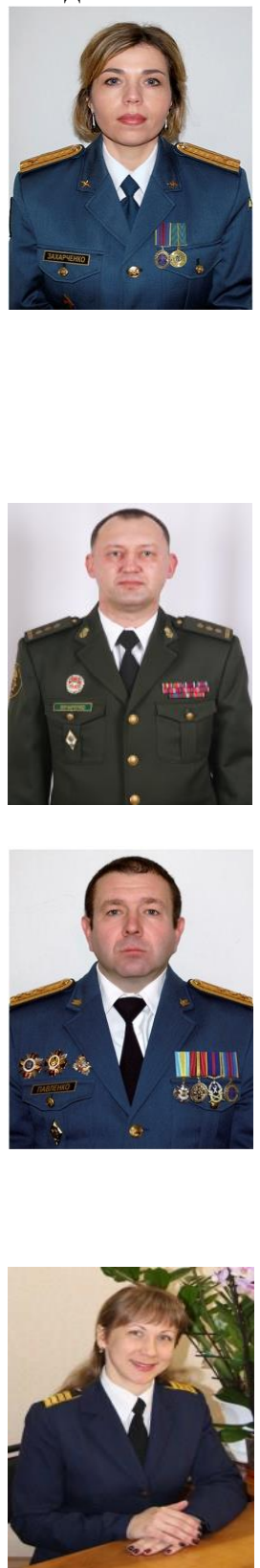

\section{Крыжевская Екатери-}

кандидат педагогических наук, доцент, доцент кафедры, Летная академия Национального авиационного университета. E-mail: kasenka01@yahoo.com на Владимировна,

\author{
Захарченко Ирина
Викторовна, кандидат \\ технических наук, \\ старший преподаватель \\ кафедры, Харьковский \\ национальный \\ университет Воздушных \\ Сил имени Ивана \\ Кожедуба \\ E-mail: \\ irishka310379@gmail.com
}

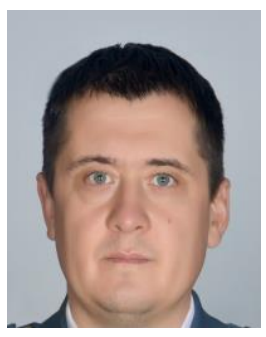

\section{Дмитриев}

Андрей

Геннадиевич

кандидат технических наук, старший научный сотрудник, начальник кафедры, Харьковский национальный

университет Воздушных Сил имени Ивана Кожедуба

E-mail:

syperdmitr@gmail.com

Овчаренко Вячеслав Владимирович, доктор военных наук, доцент, начальник факультета, Национальная академия Национальной гвардии Украины. E-mail: gepard50@gmail.com

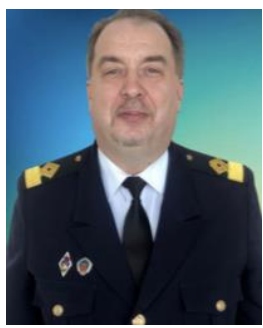

Маслов Игорь Захарович кандидат технических наук, доцент - заведующий кафедрой, Одесская морская академия E-mail: igormslv@ukr.net

\section{Павленко Максим}

Анатольевич, доктор технических наук, профессор, начальник кафедры, Харьковский национальный

университет Воздушных Сил имени Ивана Кожедуба

E-mail: bpgpma@ukr.net

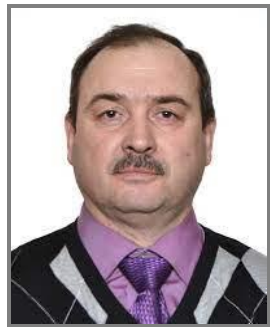

\section{Тимочко Александр} Иванович, доктор технических наук, профессор, профессор кафедры, Харьковский национальный университет Воздушных сил

E-mail:

timochko.alex@gmail.com 